\title{
A Prospective Study on Pattern of Adverse Drug Reactions to Antibiotics Commonly Prescribed In General Medicine Department of Tertiary Care Teaching Hospital
}

\author{
V. Brahma Naidu ${ }^{1}$, B. Tharun ${ }^{1}$ \\ ${ }^{1,2}$ Internee, Government General Hospital, Guntur, Andhra Pradesh, India
}

\begin{abstract}
Adverse Drug Reactions (ADRs) are an important cause of mortality and morbidity and constitute an enormous burden on the society. Many studies have implicated that the antibiotics are among the major group of drugs, which cause adverse drug reactions (ADRs). A prospective observational study was carried out in the Department of General Medicine in a 1000-bedded tertiary care teaching hospital and the The data for the study were taken from case sheets, investigation reports of patients. The causality assessment of the reported ADRs was carried out using the WHO causality assessment scale. In the WHO causality assessment scale, the drug reaction can be classified as certain, probable, possible, unlikely, and unclassified. 1025 patients were included from general medicine department. The incidence rate of antibiotic Adverse Drug Reactions was found to be $0.6 \%$. A total of 157 ADRs were collected, tabulated in CDSCO forms, analyzed and assessed on WHO causality assessment scale. Maximum ADRs were reported with cephalosporins (ceftriaxone, cifotaxim, cefixim) 50 (32\%) followed by Quinolones (Ofloxacin, Levofloxacin) 20(32\%), Penicillins 28(18\%), Aminoglycosides (Amikacin, Streptomycin, 19(12\%), Macrolides (Azithromycin) 12(8\%), and others16 (10\%). The WHO causality assessment scale was proved that $19 \%$ of ADRs are certain, $42 \%$ were probable, $29 \%$ were possible and $10 \%$ comes under unlikely and unclassified. The mortality rate was $3 \%, 23 \%$ patients needed prolonged hospital stay, and remaining patients recovered immediately. Early detection, evaluation and monitoring of ADR are essential to reduce harm to patients and thus improve public health.
\end{abstract}

Keywords: Antibiotics, Adverse drug reactions, WHO causality assessment scale

\section{Introduction}

Drugs are the commonest medical interventions, used to relieve sufferings but drugs themselves can prove fatal and result in ADR; Adverse Drug Reactions (ADRs) are an important cause of mortality and morbidity and constitute an enormous burden on the society ${ }^{1}$. Many studies have implicated that the antibiotics are among the major group of drugs, which cause adverse drug reactions (ADRs) ${ }^{1}$.

Since new drugs are being introduced every year, hence need for an active surveillance system. This is the basis for starting the international drug monitoring program by the $\mathrm{WHO}^{5}$. WHO defines ADR as " any response to a drug which is noxious and unintended, and occurs at doses normally used in man for prophylaxis, diagnosis or therapy of disease, or for the modification of physiologic function". Adverse Drug Reactions (ADRs) are important causes of mortality and morbidity. Early detection, evaluation, monitoring and reporting of ADR are essential to make drug treatment safe, efficacious and cost effective ${ }^{2}$.

Antibiotics are currently the most commonly prescribed drugs in hospitals, worldwide.The rational use of antibiotics is a major health need. Prevention of ADRs is possible by proper monitoring, which fortified the national directive to institutionalize a pharmacovigilance center in every medical college in the country. Adverse reactions are recognized hazards of drug therapy ${ }^{3}$.

Adverse Drug Reactions (ADRS) are important causes of mortality and morbidity in both hospitalized and ambulatory patients. In many countries ADRs rank among the top 10 leading causes of mortality. So there is a need to study ADRs seriously to create awareness about ADRs among patients to motivate health care professionals in the hospital to report ADRs to minimize the risk. Early detection, evaluation and monitoring of ADR are essential to reduce harm to patients and thus improve public health ${ }^{6}$

\section{Methodology}

A prospective observational study was carried out in the Department of General Medicine in a 1000-bedded tertiary care teaching hospital, A.P, India. ADRs with antibiotics were reported by both health care professional and by spontaneous reporting and analyzed using Microsoft Excel. High incidence of Antibiotics prescription and associated ADRs were observed. All patients of either sex and of any age who developed ADR were included in the study. The patients with complications, severe and terminally ill patients and emergency patients were excluded from the study. Patient case notes/files and CDSCO (suspected ADR) forms were used as main sources of data collection.

The data for the study were taken from case sheets, investigation reports of patients who had experienced an ADR, personal interviews with reporting persons or clinicians, personal interviews with patient or patient's attendant, past history of medication use, which were generally obtained from, prescriptions from the past, reports of Medical and surgical interventions, referral letters, etc. 


\section{International Journal of Science and Research (IJSR) \\ ISSN (Online): 2319-7064 \\ Index Copernicus Value (2013): 6.14 | Impact Factor (2014): 5.611}

The causality assessment of the reported ADRs was carried out using the WHO causality assessment scale. In the WHO causality assessment scale, the drug reaction can be classified as certain, probable, possible, unlikely, and unclassified.

\section{Results}

1025 patients were included from general medicine department. Out of 1025 patients, 502 were males and 523 were female. A total of 29 antibiotics were used in general medicine department. $n=799(78 \%)$ of patients admitted in general medicine were prescribed with antibiotics among them $42 \%$ were irrationally prescribed i.e. antibiotics were prescribed without any indication. Majority of the patients prescribed with antibiotics were from 41-60 age group $n=411(51.45 \%)$ followed by age 60 above group, $n=251$ (31.41\%) and least from age 18-40 year group, $n=137(17.1 \%)$ [Fig.1]. But the incidence of ADRs were found to be higher in patients of $>60$ year age group, followed by 41-60 year age group, and lowest in 18-40 year group. The age wise distribution of ADRs revealed patients of $>60$ year age group accounted $n=47(59 \%)$ followed by 41-60years age group patients $n=34(43 \%)$ and age $18-40$ year group $n=19(24 \%)$ [Fig.2].
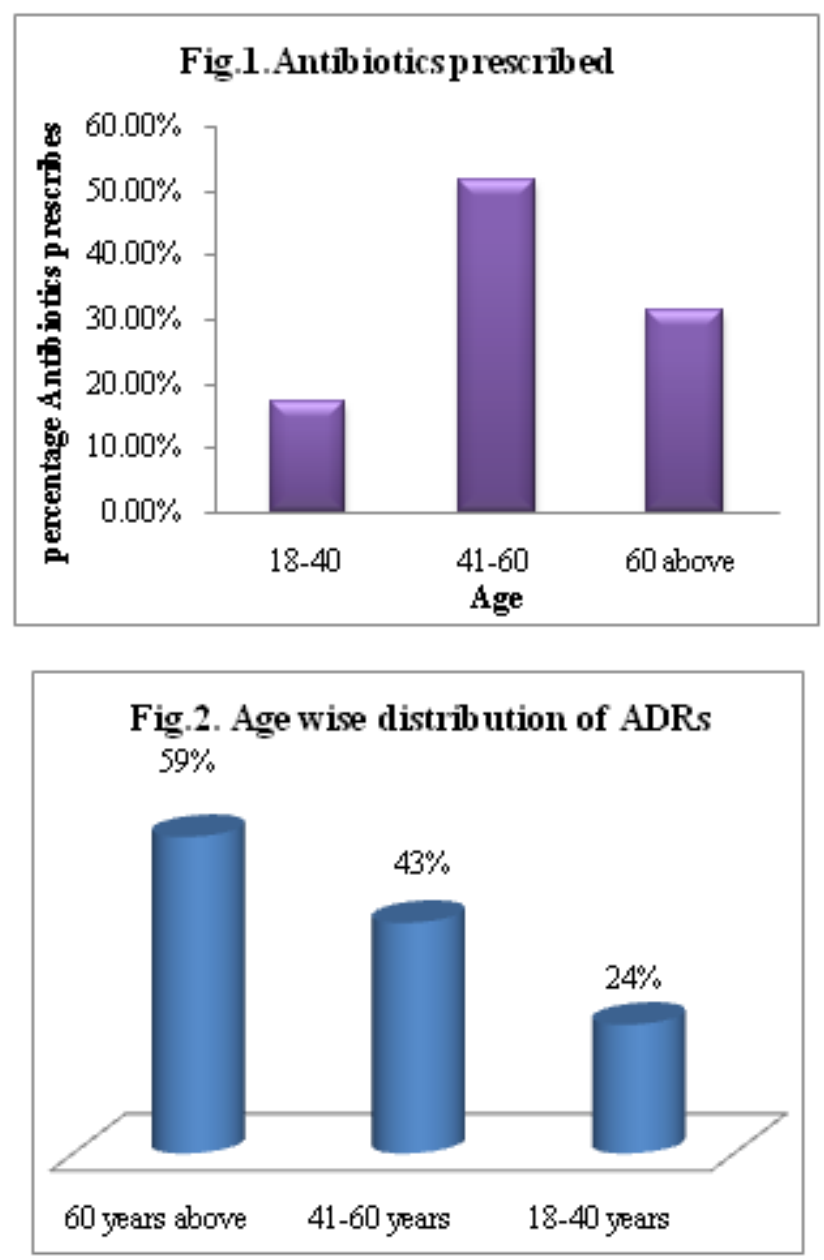

The incidence rate of antibiotic Adverse Drug Reactions was found to be $0.6 \%$. The usage of antibiotic classes is cephalosporins were the most accounted antibiotic class $32 \%$ followed by penicillins $24 \%$, fluoroquinolones $18 \%$, aminoglycosides $11 \%$, others $9 \%$ and macrolides $6 \%$ [Fig.3].

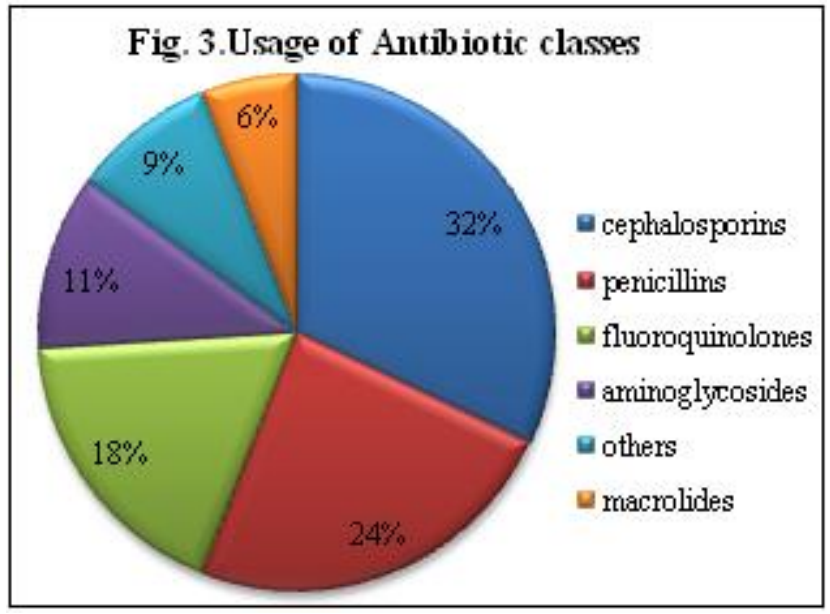

A total of 157 ADRs were collected, tabulated in CDSCO forms, analyzed and assessed on WHO causality assessment scale. Study revealed that female patients 91(58\%) predominated over males 66 (42\%) in ADR occurrence [Fig.4]

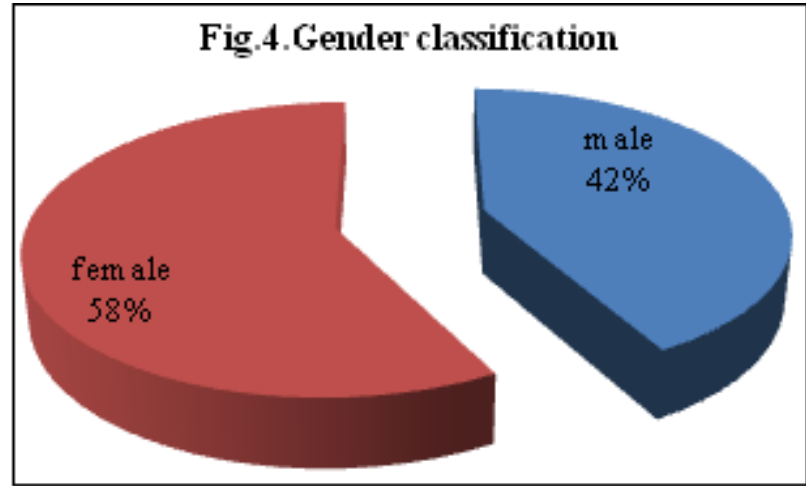

Maximum ADRs were reported with cephalosporins (ceftriaxone, cifotaxim, cefixim) 50 (32\%) followed by Quinolones (Ofloxacin, Levofloxacin) 20(32\%), Penicillins 28(18\%), Aminoglycosides (Amikacin, Streptomycin, 19(12\%), Macrolides (Azithromycin) 12(8\%), and others16 (10\%) [Fig.5].

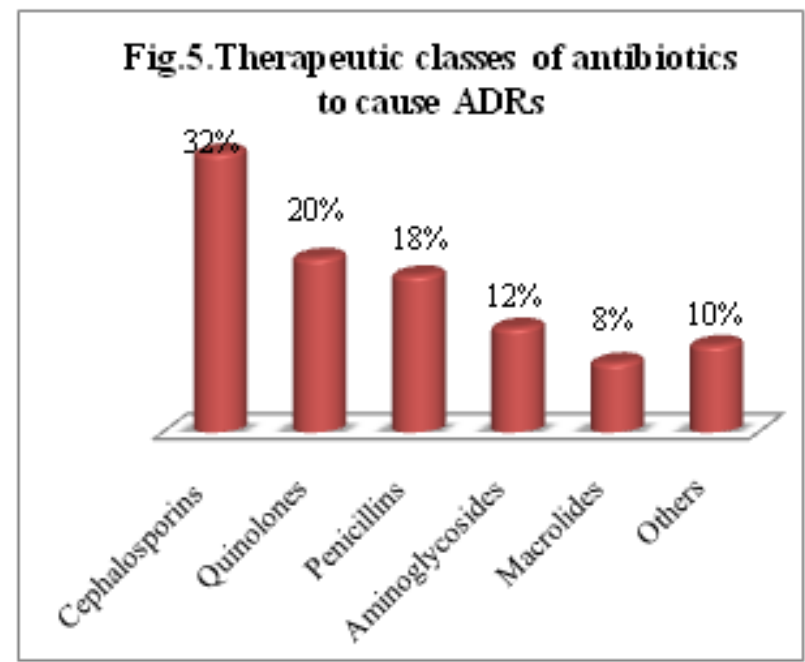




\section{International Journal of Science and Research (IJSR) \\ ISSN (Online): 2319-7064 \\ Index Copernicus Value (2013): 6.14 | Impact Factor (2014): 5.611}

The WHO causality assessment scale was proved that $19 \%$ of ADRs are certain, $42 \%$ were probable, $29 \%$ were possible and $10 \%$ comes under unlikely and unclassified. Whenever the patient had severe majority of the patients recovered from ADR [Fig.6.]. The mortality rate was 3\%, 23\% patients needed prolonged hospital stay, and remaining patients recovered immediately [Fig.7].
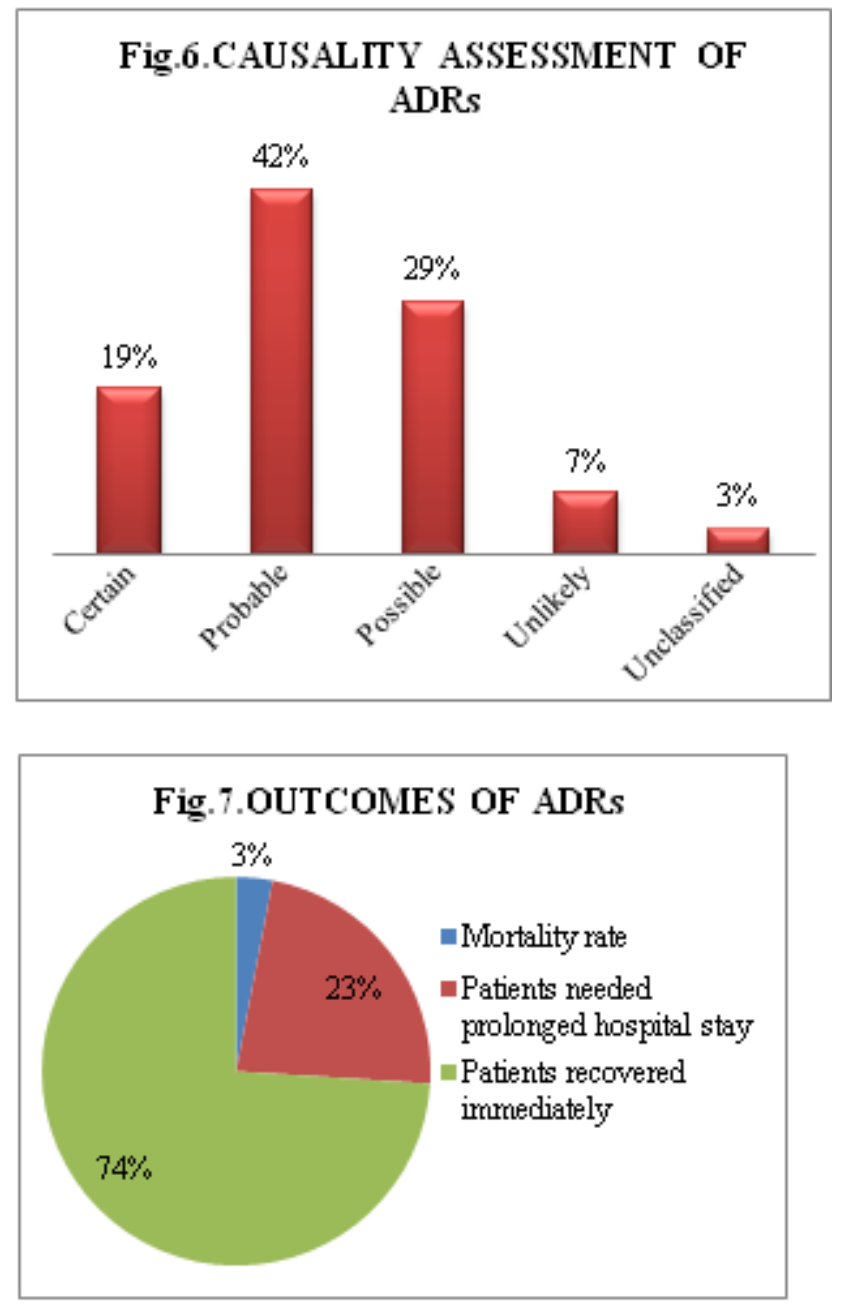

\section{Discussion}

Antibiotics are used for treatment and prophylaxis of various infectious conditions and are considered as safer drugs when used rationally. But, as with other drugs, they too show Adverse Drug Reactions. This study tried to find out the pattern of Adverse Drug Reactions of antibiotic drug class in the post marketing surveillance studies to find out the effects in a large and diverse population. In the studies carried out in Nigerian children antibiotics were the most accounted drug class in ADR occurrence and they were the second most accounted in another study (Chambers, 2006; Oshikoyaet al., 2007; Misbahet al., 2010). Antibiotics are considered as the second most prescribed drugs in the world, only next to the drugs indicated for cardiovascular diseases (Tungeret al., 2000).

In this study, the predominance of male sex for Adverse Drug Reactions may be due to majority of the admitted patients were male with more antibiotic use during the study period. Analysis of the age wise distribution showed the predominance of geriatric patients followed by adults and this result implied that the geriatric patients were more prone to antibiotic Adverse Drug Reactions due to age related pharmacokinetic and pharmacodynamic changes and the presence of co-morbid illnesses and multiple drugs along with infectious diseases. The study conducted by Jimmy Jose et al. and Suthar and Desai also showed the male predominance but the age group most accounted were adults in both the studies whereas two other studies by Starveva et al. and Hussain et al. showed adult age group and female predominance (Jose, 2008; J.V et al., 2011; Stavreva et al., 2008; Mohammed Misbah et al., 2010).

The cephalosporins were the most used antibiotic class, as shown by another study (Misbahet al., 2010) also which revealed the predominance of Cephalosporins,Vancomycin and Penicillins (Chambers et al., 2006). The analysis of the fate of the suspected drugs showed that the drug was withdrawn in many of the cases and the dose altered in some while no change was made in others keeping the risk benefit ratio consideration .In some cases the use of antibiotic was according to the culture and sensitivity reports. Drug rechallenge was not done in any of the cases. Some of the healthcare providers were not much aware of the importance of reporting and follow up of those reactions and they were not willing to change the drug or alter the dose. Vast majority of the patients recovered from ADR because none of the reported reactions were fatal (Available at http://www.WHOUMC. org/graphics/4409.pdf, Accessed on 04 November, 2014). It was also found that moderate and mild reactions were more but some severe reactions were also reported.

\section{Conclusion}

Of the 799 patients prescribed with antibiotics analyzed, it was observed that irrational antibiotics prescription is most common in general medicine department. Antibiotics comprise the major volume of the prescriptions and thus are the most irrationally prescribed drug class. So implementation of antibiotic guidelines for the hospital scenario and strict adherence should be ensured to promote the rational use.

The health system should promote the spontaneous detection of the Adverse Drug Reactions and delivering the awareness classes for the healthcare professionals regarding the need for reporting the incidents, incorporating ADR information in patient charts to improve the scenario in under-reported hospitals. Reporting of Adverse Drug Reactions to antibiotics and other drugs, proper documentation and periodic reporting to regional pharmacovigilance centers will ensure Antibiotics safety.

Pharmacovigilanceneeds to be enforced in our country for better and safe use of drugs. Although it would be prudent to initially focus on the more serious ADRs, yet it is important to consider even so called non-serious ADRs as they can have a significant impact on the patient's quality of life. The active involvement of a well trained clinical pharmacist for detecting the Adverse Drug Reactions and delivering the awareness classes for the healthcare professionals regarding 


\section{International Journal of Science and Research (IJSR) \\ ISSN (Online): 2319-7064}

Index Copernicus Value (2013): 6.14 | Impact Factor (2014): 5.611

the need of reporting the incident could improve the scenario in under-reported hospitals.

\section{References}

[1] Mohammed MisbahHussain, KundlikGirhepunje, Ranju Pal, ShahinaSugraSiddiqua. Incidence of adverse drug reactions in a tertiary care hospital: a systematic review and meta-analysis of prospective studies. Der Pharmacia Lettre, 2010;2(3): 358-368.

[2] Oshikoya KA, Njokanma OF, Chukwura HA, and Ojo IO. Adverse drug reactions in Nigerian children Paediatr. Perinat. Drug Ther., 2007; 8:81-88.

[3] Jose Jimmy, Rao Padma G.M., Jimmy Beena. Adverse drug reactions to fluoroquinolone antibiotics - analysis of reports received in a tertiary care hospital. Int. J. Risk Saf. Med. 2008;20:169-180.

[4] Mohammed MisbahHussain, KundlikGirhepunje, Ranju Pal, ShahinaSugraSiddiqua. Incidence of adverse drug reactions in a tertiary care hospital: a systematic review and meta-analysis of prospective studies. Der Pharmacia Lettre. 2010;2(3):358-368.

[5] The use of the WHO-UMC system for standardised case causality assessment. Available at :http://www.WHOUMC.org/ graphics/4409.pdf [Accessed on 8th October 2014].

[6] Stavreva G., Pendicheva D., Pandurska A., Marev R. Detection of adverse drug reactions to antimicrobial drugs in hospitalized patients. Trakia J. Sci. 2008;6(1):79. 